\title{
Highly sensitive refractive index sensor by floating nano-particles in the solution for the detection of glucose/fructose concentration
}

Hamid Bahador ( $\square$ hamid.bahador@uma.ac.ir)

University of Mohaghegh Ardabili https://orcid.org/0000-0002-5038-5901

Hamid Heidarzadeh

University of Mohaghegh Ardabili

\section{Research Article}

Keywords: Plasmonic, nano-particles, meshed SiO2, sensitivity, refractive index sensor

Posted Date: April 22nd, 2021

DOl: https://doi.org/10.21203/rs.3.rs-397841/v1

License: (c) (i) This work is licensed under a Creative Commons Attribution 4.0 International License.

Read Full License

Version of Record: A version of this preprint was published at Optical and Quantum Electronics on November 13th, 2021. See the published version at https://doi.org/10.1007/s11082-021-03334-8. 
Highly sensitive refractive index sensor by floating nano-particles in the solution for the detection of glucose/fructose concentration

\author{
Hamid Bahador ${ }^{1, *}$, Hamid Heidarzadeh ${ }^{1}$ \\ ${ }^{1}$ Department of Electrical and Computer Engineering, University of Mohaghegh Ardabili, \\ Ardabil, Iran.
}

*Corresponding author: hamid.bahador@uma.ac.ir

\begin{abstract}
In this research, an optimum design for better usage of field resonance was proposed. More interaction of the field that formed around nano-particles with a solution which has refractive index variations substantially can increase the sensitivity of the plasmonic biosensors. More interaction could be provided by floating nano-particles in the solution. The proposed structure, which has peak plasmonic resonance, has better light sensing features in comparison to similar non-floating structures. While without floating nano-particle, sensitivity is $265.8 \mathrm{~nm} / \mathrm{RIU}$ with the realization of the full floating nano-particles, the sensitivity of the structure with elliptic disc and circular disc nano-particles are $574.2 \mathrm{~nm} / \mathrm{RIU}$ and $425 \mathrm{~nm} / \mathrm{RIU}$, respectively. FWHM (Full width at half maximum) of the structure with elliptic disc and circular disc nano-particles are $57.4 \mathrm{~nm}$ and 49.4 $\mathrm{nm}$, respectively. In presence of meshed $\mathrm{SiO}_{2}$ network and pseudo-floating form, sensitivity and FWHM of the structure are $504 \mathrm{~nm} /$ RIU and $58.4 \mathrm{~nm}$ for elliptic discs and $372 \mathrm{~nm} / \mathrm{RIU}$ and 49.9 $\mathrm{nm}$ for circular discs, respectively.
\end{abstract}

Keywords: Plasmonic; nano-particles; meshed $\mathrm{SiO}_{2}$; sensitivity; refractive index sensor; 


\section{Introduction}

Plasmonic nanostructures able to control light in space smaller than the diffraction limit, have a widespread application in waveguides, detectors[1-3], solar cells[4-8], and lasers [9]. Based on the precise permeability function of metals, their optical properties are inherently dependent on the fluctuating behavior of electrons in the metal. The result of electron-photon interaction in a metal nanostructure is localized surface plasmons. These non-propagation excitations are produced from the scattering of the oscillating electromagnetic waves by a small conductive nanoparticle in the sub-wavelength band. The realization of the oscillation condition depends on, shape, size, the material of metal nanoparticle, and distance between them and the surrounding environment[10-14]. In the past decades, a variety of biosensors have been developed that incorporate plasmonic nanoparticles $[15,16]$. The small-sized and bio-compatible sensing sensors are the critical elements to realize the best nano biosensors [17]. Surface Plasmon Resonance (SPR) is a physical optical phenomenon that exists at the interface between metal and dielectric [18, 19]. When light is incident on the interface between metal and dielectric, the evanescent wave when total internal reflection occurs causes free electrons in the metal to generate surface plasmon. After proper adjustment, when the light coupled into the metal film resonates with the surface plasmon unit, part of the energy of the incident light will be converted into the energy of the surface plasmon unit, forming a surface plasmon resonance. When the refractive index of the substance adsorbed on the metal film surface is different, the resonance peak position will be different. So, the refractive index is an important physical quantity reflecting the interaction between light and medium. Based on the sensitive response of surface plasmons to changes in the refractive index of the surrounding environment. The working principle of most optical biosensors is to adsorb biological substances to the surface of the device and measure the change in the refractive index of the substance. Surface plasmon resonance has become the basis of existing biosensors, although the resulting detectors are usually too large and rely too much on moving parts to be incorporated into portable digital devices. As a result, scientists began to study plasmonic micro and nanosensors as a possible compact configuration for next-generation biosensors [20]. The plasmon based nano-bio-sensors are becoming an ultra-sensitive chemical and biological analysis method [21]. The overall quality factor or figure of merit (FoM) of the LSP-based nano-sensors sensing response is severely affected by the wide local plasmon resonance linewidth due to the large inherent absorption of general metals and the main radiation scattering contribution $[22,23]$. To increase the total FoM, some efforts have been made to enhance the refractive index sensitivity of individual plasmonic nanoparticles by optimizing their size and shape [24-26]. Recently, several new design principles have been proposed, including plasmonic hybridization [27], transformation optics [28], and subgroup decomposition [29], which are used to modify the resonance line shape and enhance the spectral contrast of individual metal nanodimers and nanoclusters. However, the reduction of the LSP resonance linewidth based on the aforementioned strategy is very 
limited, and the quality factors of the hybrid resonances are still about an order of magnitude smaller than the quality factors of the propagating plasma. All of the above methods usually rely on strict design and precise control of the arrangement of individual components, such as the distance between particles and particle shape, which further causes experimental difficulties (especially in manufacturing) and cannot be applied to the following components. Optimized usage of field resonance around nanoparticle could increase the sensitivity of the detector. Floating of metal nanoparticle in solution could increase interaction filed produced around particle and solution. In this work, the floating of nanoparticle at an appropriate distance from the substrate and its effect on detector performance improvement is investigated. The effect of geometric dimensions of floating nanoparticle and its gap from the substrate for the realization of strong field and proper interaction will be investigated. A proper structure for a floating particle, which can be fabricated, has been proposed.

\section{Proposed Structure}

Increasing the volume of the field interaction with the solution can improve the sensitivity of the plasmonic structure. As mentioned in reference [30], the shift in the wave vector, $k$, is proportional to the overlap integral, which in turn is proportional to the volume of the interaction, $\mathrm{V}_{\text {in }}$ :

$$
\delta k \approx \frac{k_{i}}{2} \frac{\int_{V_{i n}} \delta \varepsilon \cdot \overrightarrow{E_{i}^{*}} \cdot \overrightarrow{E_{f}} \cdot d r}{\int_{V} \varepsilon \cdot \overrightarrow{E_{i}^{*}} \cdot \overrightarrow{E_{f}} \cdot d r}
$$

Where $E_{i}$ and $k_{i}$ are the electric field and its wave vector before the change in the refractive index of the solution, whereas, $\mathrm{E}_{\mathrm{f}}$ is field after variation of refractive index and $\delta k$ is the corresponding shift at the wave vector due to the change in the solution dielectric constant from $\varepsilon$ to $\varepsilon+\partial \varepsilon$. Because $\delta k$ expresses the change in wavelength, $\delta k / \partial \varepsilon$ represents the sensitivity of the sensor, which is proportional to the overlap integral in the numerator of equation (1) normalized to the total energy. Therefore, to maximize the sensitivity, it is necessary to maximize this integral, which can be achieved by increasing the volume of interaction in the solution.

Floating of the particle at a specific height produces an appropriate place under the particle for more mutual effect between the solution and resulted from the field. Increasing interaction between field and solution could improve the sensitivity of the system to the refractive index of the solution. In Figure 1, a structure for pseudo-floating and increasing interaction of field and solution is demonstrated. In this design, by meshing a part of $\mathrm{SiO}_{2}$ under a particle, the solution exists under the particle, too. The sensor structure can be divided into several parts: $\mathrm{SiO}_{2}$ part grown on $\mathrm{Si}$, meshed $\mathrm{SiO}_{2}$ and metal particles above that, and finally, solution enclosing particles and meshed part. For further investigation of the structure, elliptic disc, and 
circular disc particles, all silver is used. At first, by omitting the meshed region, the effect of the full floating of the particle on sensitivity is investigated. Then the effect of meshed $\mathrm{SiO}_{2}$ area under particle is evaluated. By changing particle dimensions, a deeper understanding of the floating effect on structure, performance optimization could be achieved. For precise evaluation and comparison of system performance in different cases, the Maxwell equation is solved by finite difference time domain (FDTD), and the extinction spectra of the structure are calculated. In all simulation profiles, mesh size around nanoparticles is $\mathrm{dx}=\mathrm{dy}=\mathrm{dz}=0.1 \mathrm{~nm}$, and Johnson Christy dielectric constant of metal are used [31]. For upper and lower sides, perfectly matched layer boundary conditions, and for other sides, periodic boundary conditions are used.

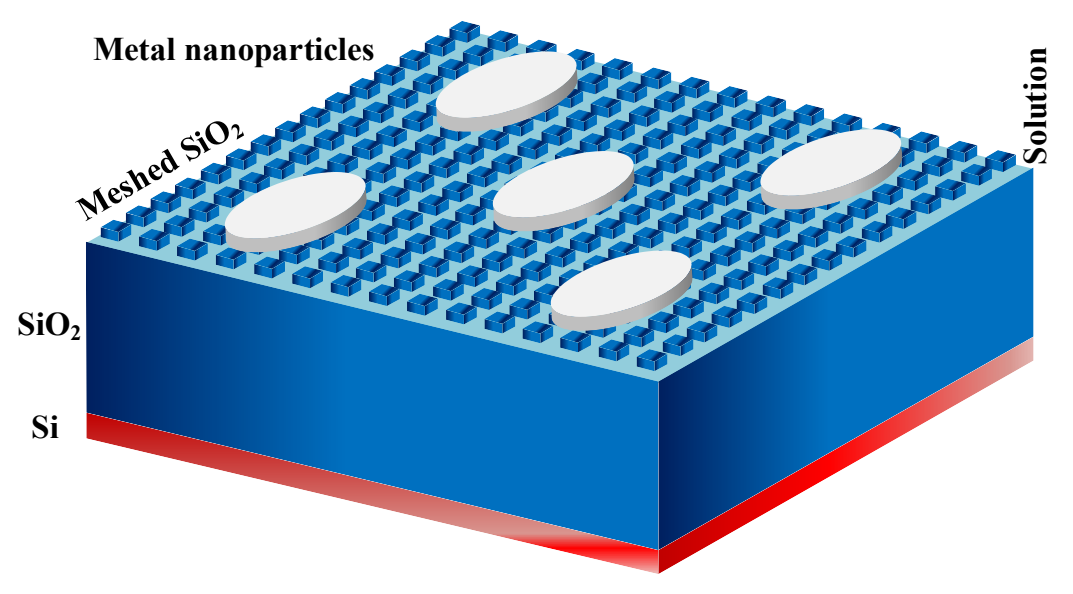

Figure 1. Sensor structure: $\mathrm{SiO}_{2}$ part grown on $\mathrm{Si}$, meshed $\mathrm{SiO}_{2}$ and metal particles above it, solution enclosing particles and meshed part.

\section{Results and discussion}

Variation of the refractive index of solution around metal nanoparticle could be considered as a shift of resonance wavelength location. Figure 2 shows simulation results for variation of extinction spectrum of nanostructure for the case that metal particle is on the substrate and when there is a gap between the particle and substrate. This experiment is for the Ag elliptic disc with $116 \mathrm{~nm}$ large radius, $58 \mathrm{~nm}$ small radius, and $25 \mathrm{~nm}$ thickness and a solution with 1.333 refractive indexes. Sensitivity and FWHM of the structure have been reported in three cases for $\Delta \mathrm{n}=0.05$, the variation of the refractive index of the solution, in Table 1 . Results show that making the proper distance between a metal nanoparticle and the substrate increases system FOM from 4.7 to 10. As stated in this Table, with meshed $\mathrm{SiO}_{2}$ region, to realize pseudo-floating of the particle, the figure of merit and sensitivity of system with $13 \%$ reduction are 8.6 and $504.5 \mathrm{~nm} / \mathrm{RIU}$, respectively. These features show that this design improves detector sensitivity at least twice. Figure 3 shows the electrical field profile, which is formed under particle. The existence of this strong field and 
oscillation in the region in which a solution exists improves the sensitivity of the detector to the refractive index of the solution, considerably.

The effect of the gap between a metal nanoparticle and substrate to form a strong field that has good interaction with the solution has been illustrated in Figure 4. The effect of gap change for the Ag elliptic disc on the extinction spectrum is shown in Figure 4.a. Sensitivity variation and normalized amplitude of the extinction spectrum peak is also shown in Figure 4.b. This elliptic nanoparticle has a large radius of 116 $\mathrm{nm}$, a small radius of $58 \mathrm{~nm}$, and a thickness of $25 \mathrm{~nm}$.

Figure 4.c shows the variation trend of the extinction spectrum of the Ag circular disc with the variation of the gap between particle and substrate. This disc has a radius of $116 \mathrm{~nm}$ and a thickness of $30 \mathrm{~nm}$. Also, Figure 4.d shows the sensitivity and amplitude of the extinction spectrum as a function of the gap. Sensitivity and FWHM of the structure for Ag circular disks have been reported in Table 2. To have both good sensitivity and proper amplitude of the extinction spectrum, a $70 \mathrm{~nm}$ gap for the elliptic disc and 40 $\mathrm{nm}$ for the circular disc has been used.

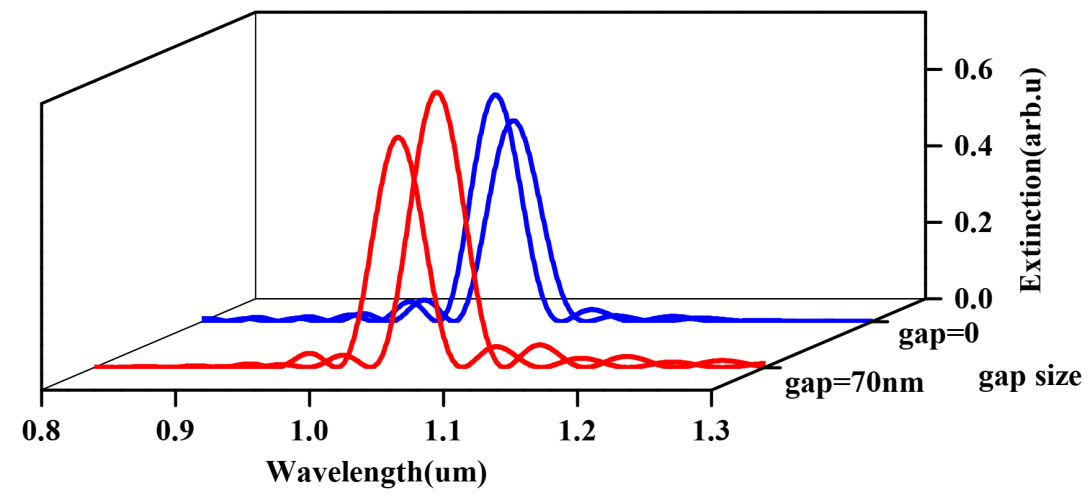

Figure 2. Variation of extinction spectrum of nanostructure without the gap between the substrate and nanoparticles $(\mathrm{gap}=0)$ and with the gap $(\mathrm{gap}=70 \mathrm{~nm})$

Table 1. Sensitivity and FWHM of the structure for elliptic disks for three cases: gap=0, gap=70 without meshed $\mathrm{SiO}_{2}$, and gap=70 with meshed $\mathrm{SiO}_{2}$

\begin{tabular}{ccccc}
\hline gap size $(\mathbf{n m})$ & $\begin{array}{c}\text { sensitivity } \\
(\mathbf{n m} / \mathbf{R I U})\end{array}$ & $\begin{array}{c}\text { FWHM } \\
(\mathbf{n m})\end{array}$ & $\begin{array}{c}\text { FOM } \\
(\text { S/FWHM) }\end{array}$ & $\begin{array}{c}\text { Peak intensity } \\
\text { normalized }\end{array}$ \\
\hline $\mathbf{0}$ & 265.8 & 56.3 & 4.7 & 0.59 \\
\hline $\mathbf{7 0}$ & 574.2 & 57.4 & 10 & 0.6 \\
\hline $\mathbf{7 0 ~ \& ~} \mathbf{m e s h}-\mathbf{S i O}_{2}$ & 504.5 & 58.4 & 8.6 & 0.66 \\
\hline
\end{tabular}




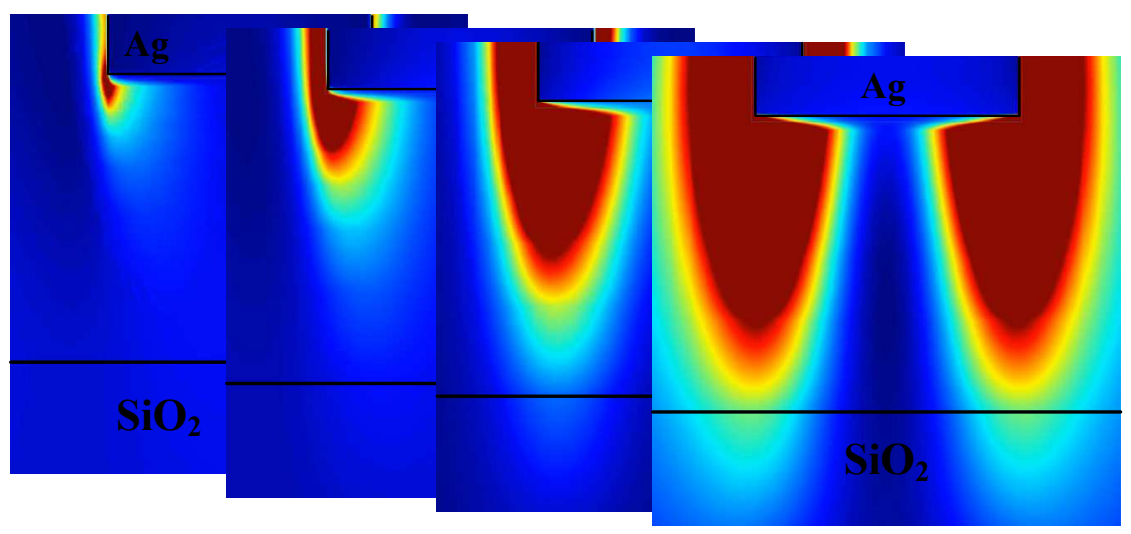

Figure 3. Electrical field profile, which is formed under elliptic nanoparticle.

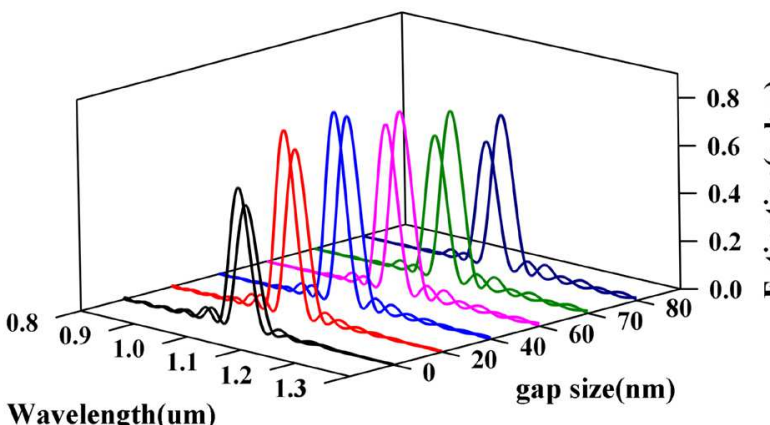

Wavelength(um)

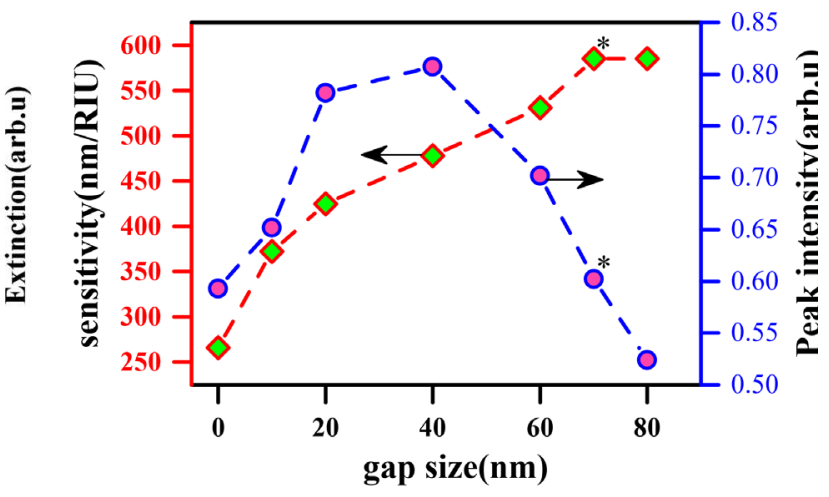

(b)

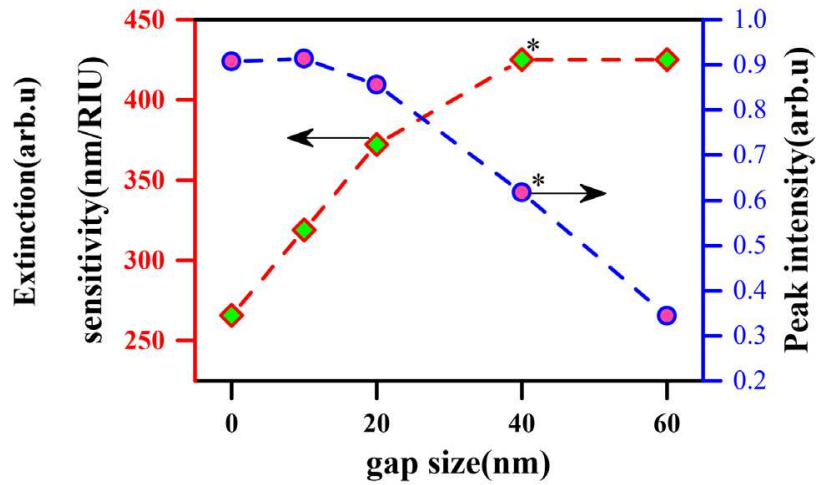

(d)

Figure 4. (a) The effect of gap variation for the Ag elliptic disc on extinction spectrum and (b) Sensitivity variation and normalized amplitude of extinction spectrum peak (c) The effect of gap change for the Ag circular disc on extinction spectrum and (d) Sensitivity variation and normalized amplitude of extinction spectrum peak.

Increasing the radius from 96 to $126 \mathrm{~nm}$ reduces system sensitivity from 425.3 to $372.1 \mathrm{~nm} / \mathrm{RIU}$. Also, the FWHM of the structure increases from $44.6 \mathrm{~nm}$ to $51.6 \mathrm{~nm}$. While in these radius ranges, the normalized 
peak amplitude of the extinction spectrum is increased from 0.32 to 0.77 . It seems that $116 \mathrm{~nm}$ for the $40 \mathrm{~nm}$ gap and $30 \mathrm{~nm}$ thickness is a proper choice.

Increasing the radius of the Ag elliptic disc, from a large radius of $96 \mathrm{~nm}$ and a small radius of $48 \mathrm{~nm}$ to a large radius of $126 \mathrm{~nm}$ and a small radius of $63 \mathrm{~nm}$ produces a similar result to that of a circular disc. In this range, sensitivity and FWHM of structure change from $548 \mathrm{~nm} / \mathrm{RIU}$ and $50.05 \mathrm{~nm}$ to $531 \mathrm{~nm} / \mathrm{RIU}$ and 60.6 , respectively. Variation of the normalized peak amplitude of the extinction spectrum by increasing radius, from 0.24 to 0.8 shows that a particle with a large radius of $116 \mathrm{~nm}$, a small radius of $58 \mathrm{~nm}$ has a peak amplitude of the extinction spectrum, which could be considered an optimum size. Investigation of the relationship between changing particle radius and detector parameters has been done for a particle with a $70 \mathrm{~nm}$ gap and $25 \mathrm{~nm}$ thickness.

Table 2. Sensitivity and FWHM of the structure for circular disks for three cases: gap $=0$, gap $=40$ without meshed $\mathrm{SiO}_{2}$, and gap $=40$ with meshed $\mathrm{SiO}_{2}$

\begin{tabular}{ccccc}
\hline gap size (nm) & $\begin{array}{c}\text { sensitivity } \\
\text { (nm/RIU) }\end{array}$ & $\begin{array}{c}\text { FWHM } \\
\text { (nm) }\end{array}$ & $\begin{array}{c}\text { FOM } \\
\text { (S/FWHM) }\end{array}$ & $\begin{array}{c}\text { Peak intensity } \\
\text { normallized }\end{array}$ \\
\hline $\mathbf{0}$ & 265.8 & 48.9 & 5.4 & 0.9 \\
\hline $\mathbf{4 0}$ & 425.3 & 49.4 & 8.6 & 0.6 \\
\hline $\mathbf{4 0} \&$ mesh-SiO & 372 & 49.9 & 7.45 & 0.66 \\
\hline
\end{tabular}

Simulation results to choose a good thickness for particle show that changing the thickness of Ag circular disc does not have a considerable effect on detector parameters and $30 \mathrm{~nm}$ thickness has a good normalized peak amplitude of the extinction spectrum. Table 3, shows simulation results for thickness variation in silver elliptic disc. From Table data, it could be seen that $25 \mathrm{~nm}$ thickness is a good choice for this particle.

Table 3. The effect of thickness variation in silver elliptic disk

\begin{tabular}{|c|c|c|c|c} 
thickness $(\mathbf{n m})$ & $\begin{array}{c}\text { sensitivity } \\
\text { (nm/RIU) }\end{array}$ & $\begin{array}{c}\text { FWHM } \\
\mathbf{( n m )}\end{array}$ & $\begin{array}{c}\text { FOM } \\
\text { (S/FWHM) }\end{array}$ & $\begin{array}{c}\text { Peak intensity } \\
\text { normalized }\end{array}$ \\
$\mathbf{2 0}$ & 585 & 61.13 & 9.57 & 0.7 \\
$\mathbf{2 5}$ & 585 & 57.414 & 10.189 & 0.602 \\
$\mathbf{3 0}$ & 532 & 54.218 & 9.81 & 0.509 \\
$\mathbf{3 5}$ & 531 & 53.161 & 9.9885 & 0.431 \\
$\mathbf{4 0}$ & 584.9 & 52.096 & 11.22 & 0.36
\end{tabular}

\section{Application for glucose/fructose monitoring}

In the beverage industry, the refractive index and its variations are used to detect and measure the main components existing in aqueous solutions like ethanol, glycerol, and glucose/fructose. Because of its good resolution, a plasmonic sensor can be used to measure glucose/fructose density 
in a liquid. In [32], the value of the refractive index of a model aqueous solution containing glucose/fructose has been measure and recorded. In this reference, by the regression analysis of experimental data, the equation below has been proposed to obtain the refractive index of the liquid solution including glucose/fructose:

$$
\mathrm{n}=1.3329+0.000135 *(\text { glucose/fructose })(\mathrm{g} / \mathrm{L})
$$

With the aid of this equation, Table 4 shows the variation of the refractive index of a model aqueous solution containing glucose/fructose. To measure the quantity of glucose/fructose, the model aqueous solution is replaced in the structure of the sensor. Figure 5 shows the relocation of extinction spectrums by different densities of glucose/fructose. The peak wavelength of the extinction spectrum for different densities of glucose/fructose of $0,4,8,12$, and $16 \mathrm{mg}$ in every $100 \mathrm{~mL}$ are 1046.5, 1049.2, 1051.9, 1054.5, and $1057.2 \mathrm{~nm}$ which are spaced around $2.65 \mathrm{~nm}$ from each other. Calculating output results from Figure 5 shows that FOM and sensitivity are 8.25 and $495 \mathrm{~nm} / \mathrm{RIU}$, respectively.

Table 4. Peak wavelength as a function of densities of glucose/fructose.

\begin{tabular}{cccccc} 
Glucose/Fructose $(\mathbf{g} / \mathbf{1 0 0} \mathbf{m L})$ & $\mathbf{0}$ & $\mathbf{4}$ & $\mathbf{8}$ & $\mathbf{1 2}$ & $\mathbf{1 6}$ \\
\hline Refractive index $(\mathrm{n})$ & 1.3330 & 1.3383 & 1.3437 & 1.3491 & 1.3545 \\
\hline Peak wavelength & 1046.5 & 1049.2 & 1051.9 & 1054.5 & 1057.2
\end{tabular}

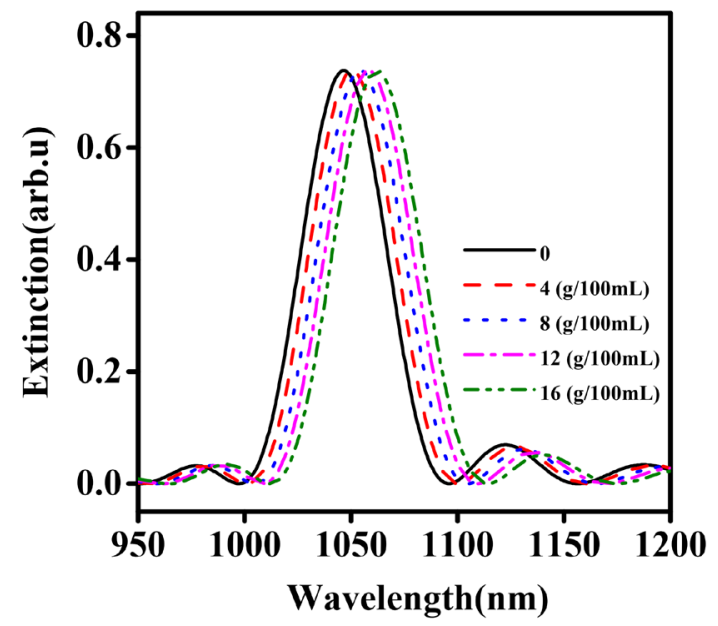

Figure 5. Extinction spectrums for different densities of glucose/fructose. 


\section{Conclusion}

In this work, the floating of nano-particles in a solution with varying refractive index has been investigated for the first time. Floating nano-particles at a specific height above the substrate improves the sensitivity of the refractive index sensor. Parameters to evaluate the sensor, such as extinction spectrums and electric field distribution, are calculated by the FDTD method. Without the floating of nano-particle, the sensitivity is calculated as $265.8 \mathrm{~nm} / \mathrm{RIU}$. Floating oval disc and circular disc nano-particles increases the sensitivity to values higher than 10 and 8.6. Also, the effect of nano-particle distance from substrate and their dimensions to achieve the best sensitivity evaluated and simulated. Finally, for the realization of floating nano-particles, a meshed structure of $\mathrm{SiO}_{2}$ is added underneath the nano-particles and its effect evaluated as a pseudo-floating case. The proposed structure and completed simulations can be used as a novel method in sensory applications for the production of very sensitive plasmonic sensors.

\section{References}

[1] H. Bahador and H. Heidarzadeh, "Analysis and Simulation of a Novel Localized Surface Plasmonic Highly Sensitive Refractive Index Sensor," Plasmonics, pp. 1-7, 2020.

[2] H. Heidarzadeh, "Analysis and simulation of a plasmonic biosensor for hemoglobin concentration detection using noble metal nano-particles resonances," Optics Communications, vol. 459, p. 124940, 2020.

[3] H. Heidarzadeh, "Highly Sensitive Plasmonic Sensor Based on Ring Shape Nanoparticles for the Detection of Ethanol and D-Glucose Concentration," IEEE Transactions on Nanotechnology, vol. 19, pp. 397-404, 2020.

[4] A. Jangjoy, H. Bahador, and H. Heidarzadeh, "Design of an ultra-thin silicon solar cell using Localized Surface Plasmonic effects of embedded paired nanoparticles," Optics Communications, vol. 450, pp. 216-221, 2019.

[5] G. Mokari and H. Heidarzadeh, "Efficiency enhancement of an ultra-thin silicon solar cell using plasmonic coupled core-shell nanoparticles," Plasmonics, vol. 14, pp. 1041-1049, 2019.

[6] F. Sobhani, H. Heidarzadeh, and H. Bahador, "Photocurrent improvement of an ultra-thin silicon solar cell using the localized surface plasmonic effect of clustering nanoparticles," Chinese Physics B, vol. 29, p. 068401, 2020.

[7] F. Sobhani, H. Heidarzadeh, and H. Bahador, "Efficiency enhancement of an ultra-thin film silicon solar cell using conical-shaped nanoparticles: similar to superposition (top, middle, and bottom)," Optical and Quantum Electronics, vol. 52, pp. 1-13, 2020.

[8] A. Jangjoy, H. Bahador, and H. J. P. Heidarzadeh, "A Comparative Study of a Novel Anti-reflective Layer to Improve the Performance of a Thin-Film GaAs Solar Cell by Embedding Plasmonic Nanoparticles," pp. 1-7, 2020.

[9] E. Cubukcu, E. A. Kort, K. B. Crozier, and F. J. A. P. L. Capasso, "Plasmonic laser antenna," vol. 89, p. 093120, 2006. 
[10] K. L. Kelly, E. Coronado, L. L. Zhao, and G. C. Schatz, "The optical properties of metal nanoparticles: the influence of size, shape, and dielectric environment," ed: ACS Publications, 2003.

[11] S. Link and M. A. El-Sayed, "Spectral properties and relaxation dynamics of surface plasmon electronic oscillations in gold and silver nanodots and nanorods," ed: ACS Publications, 1999.

[12] J. Pérez-Juste, I. Pastoriza-Santos, L. M. Liz-Marzán, and P. Mulvaney, "Gold nanorods: synthesis, characterization and applications," Coordination chemistry reviews, vol. 249, pp. 1870-1901, 2005.

[13] J. Zhang, H. Liu, Z. Wang, and N. Ming, "Shape-selective synthesis of gold nanoparticles with controlled sizes, shapes, and plasmon resonances," Advanced Functional Materials, vol. 17, pp. 3295-3303, 2007.

[14] F. Sobhani, H. Heidarzadeh, and H. J. C. P. B. Bahador, "Photocurrent improvement of an ultrathin silicon solar cell using the localized surface plasmonic effect of clustering nanoparticles," vol. 29, p. 068401, 2020.

[15] A. Belushkin, F. Yesilkoy, and H. J. A. n. Altug, "Nanoparticle-enhanced plasmonic biosensor for digital biomarker detection in a microarray," vol. 12, pp. 4453-4461, 2018.

[16] A. Vázquez-Guardado, S. Barkam, M. Peppler, A. Biswas, W. Dennis, S. Das, S. Seal, and D. J. N. 1. Chanda, "Enzyme-free plasmonic biosensor for direct detection of neurotransmitter dopamine from whole blood," vol. 19, pp. 449-454, 2018.

[17] C.-Y. Chang, H.-T. Lin, M.-S. Lai, T.-Y. Shieh, C.-C. Peng, M.-H. Shih, and Y.-C. J. S. R. Tung, "Flexible localized surface plasmon resonance sensor with metal-insulator-metal nanodisks on PDMS substrate," vol. 8, pp. 1-8, 2018.

[18] J. Homola, S. S. Yee, G. J. S. Gauglitz, and a. B. Chemical, "Surface plasmon resonance sensors," vol. 54, pp. 3-15, 1999.

[19] H. Heidarzadeh, A. Rostami, M. Dolatyari, and G. J. A. o. Rostami, "Plasmon-enhanced performance of an ultrathin silicon solar cell using metal-semiconductor core-shell hemispherical nanoparticles and metallic back grating," vol. 55, pp. 1779-1785, 2016.

[20] X. D. Hoa, A. Kirk, M. J. B. Tabrizian, and bioelectronics, "Towards integrated and sensitive surface plasmon resonance biosensors: a review of recent progress," vol. 23, pp. 151-160, 2007.

[21] X. Liu, D. Zhang, Y.-C. Wu, M. Yang, Q. Wang, C. Ó. Coileáin, H. Xu, C. Yang, M. Abid, and M. J. C. Abid, "Ultra-sensitive graphene based mid-infrared plasmonic bio-chemical sensing using dielectric beads as a medium," vol. 122, pp. 404-410, 2017.

[22] C. Huang, J. Ye, S. Wang, T. Stakenborg, and L. J. A. P. L. Lagae, "Gold nanoring as a sensitive plasmonic biosensor for on-chip DNA detection," vol. 100, p. 173114, 2012.

[23] B. Špačková, P. Wrobel, M. Bocková, and J. J. P. o. t. I. Homola, "Optical biosensors based on plasmonic nanostructures: a review," vol. 104, pp. 2380-2408, 2016.

[24] S. Zhu, H. Li, M. Yang, and S. W. J. N. Pang, "High sensitivity plasmonic biosensor based on nanoimprinted quasi 3D nanosquares for cell detection," vol. 27, p. 295101, 2016.

[25] H. J. O. C. Heidarzadeh, "Analysis and simulation of a plasmonic biosensor for hemoglobin concentration detection using noble metal nano-particles resonances," vol. 459, p. 124940, 2020.

[26] N. Amoosoltani, N. Yasrebi, A. Farmani, and A. J. I. S. J. Zarifkar, "A plasmonic nano-biosensor based on two consecutive disk resonators and unidirectional reflectionless propagation effect," 2020 . 
[27] Y. Liu, Y. Nie, M. Wang, Q. Zhang, Q. J. B. Ma, and Bioelectronics, "Distance-dependent plasmon-enhanced electrochemiluminescence biosensor based on MoS2 nanosheets," vol. 148, p. $111823,2020$.

[28] A. Fernández-Domínguez, A. Wiener, F. García-Vidal, S. Maier, and J. J. P. r. 1. Pendry, "Transformation-optics description of nonlocal effects in plasmonic nanostructures," vol. 108, p. $106802,2012$.

[29] M. Rahmani, D. Y. Lei, V. Giannini, B. Lukiyanchuk, M. Ranjbar, T. Y. F. Liew, M. Hong, and S. A. J. N. 1. Maier, "Subgroup decomposition of plasmonic resonances in hybrid oligomers: modeling the resonance lineshape," vol. 12, pp. 2101-2106, 2012.

[30] I. Abdulhalim, "Biosensing configurations using guided wave resonant structures," in Optical Waveguide Sensing and Imaging, ed: Springer, 2008, pp. 211-228.

[31] P. B. Johnson and R.-W. J. P. r. B. Christy, "Optical constants of the noble metals," vol. 6, p. 4370, 1972.

[32] A. Shehadeh, A. Evangelou, D. Kechagia, P. Tataridis, A. Chatzilazarou, and F. Shehadeh, "Effect of ethanol, glycerol, glucose/fructose and tartaric acid on the refractive index of model aqueous solutions and wine samples," Food Chemistry, p. 127085, 2020. 


\section{Figures}

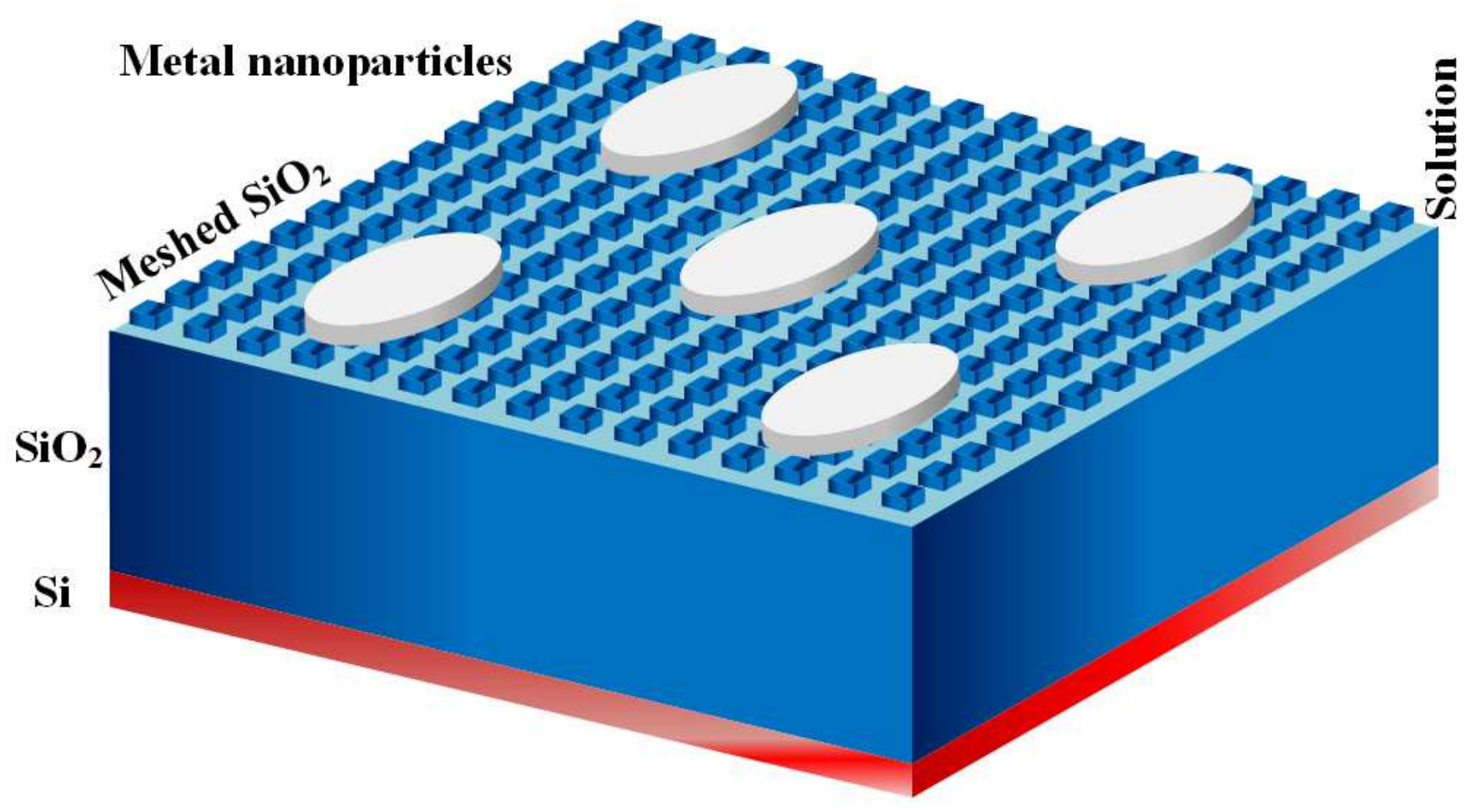

Figure 1

Sensor structure: $\mathrm{SiO} 2$ part grown on $\mathrm{Si}$, meshed $\mathrm{SiO} 2$ and metal particles above it, solution enclosing particles and meshed part.

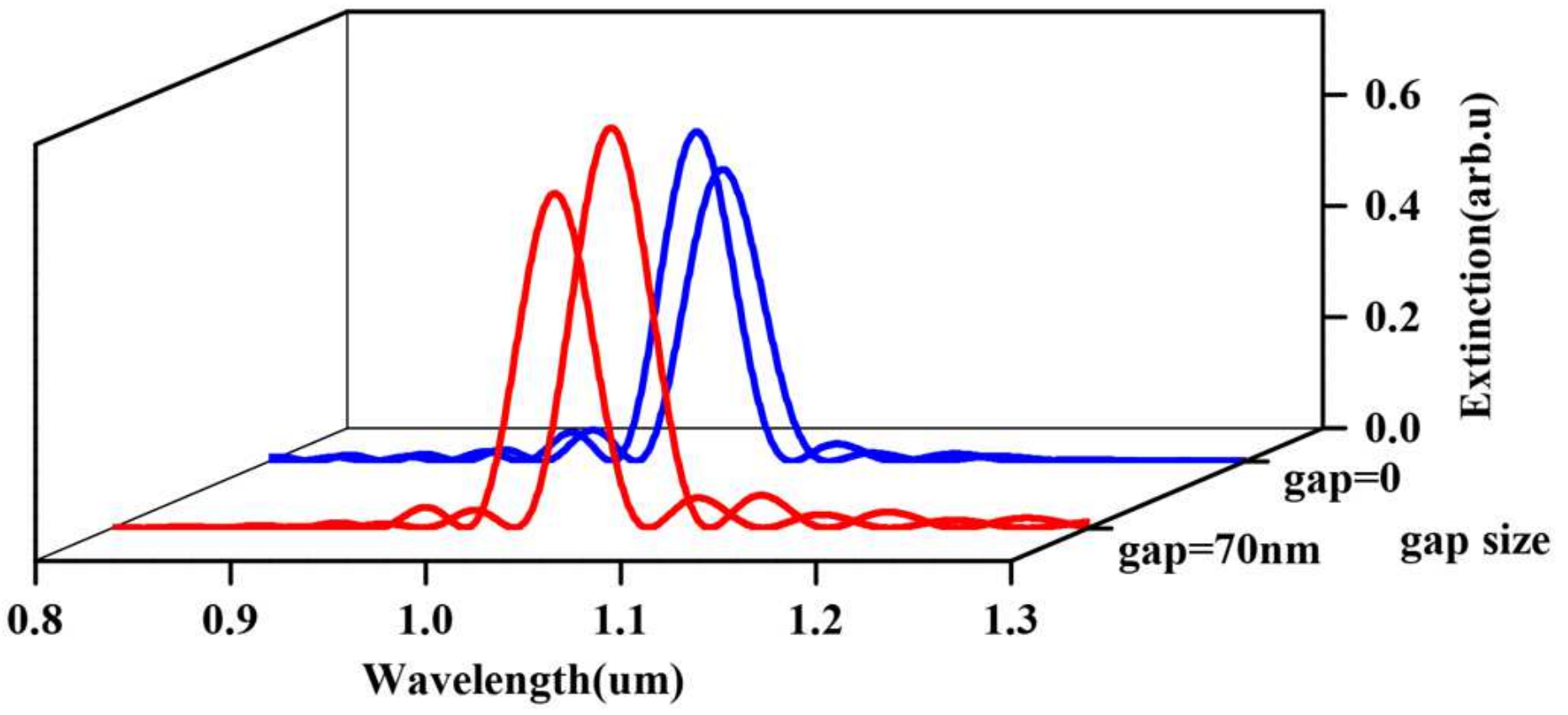


Figure 2

Variation of extinction spectrum of nanostructure without the gap between the substrate and nanoparticles (gap $=0)$ and with the gap (gap=70nm)

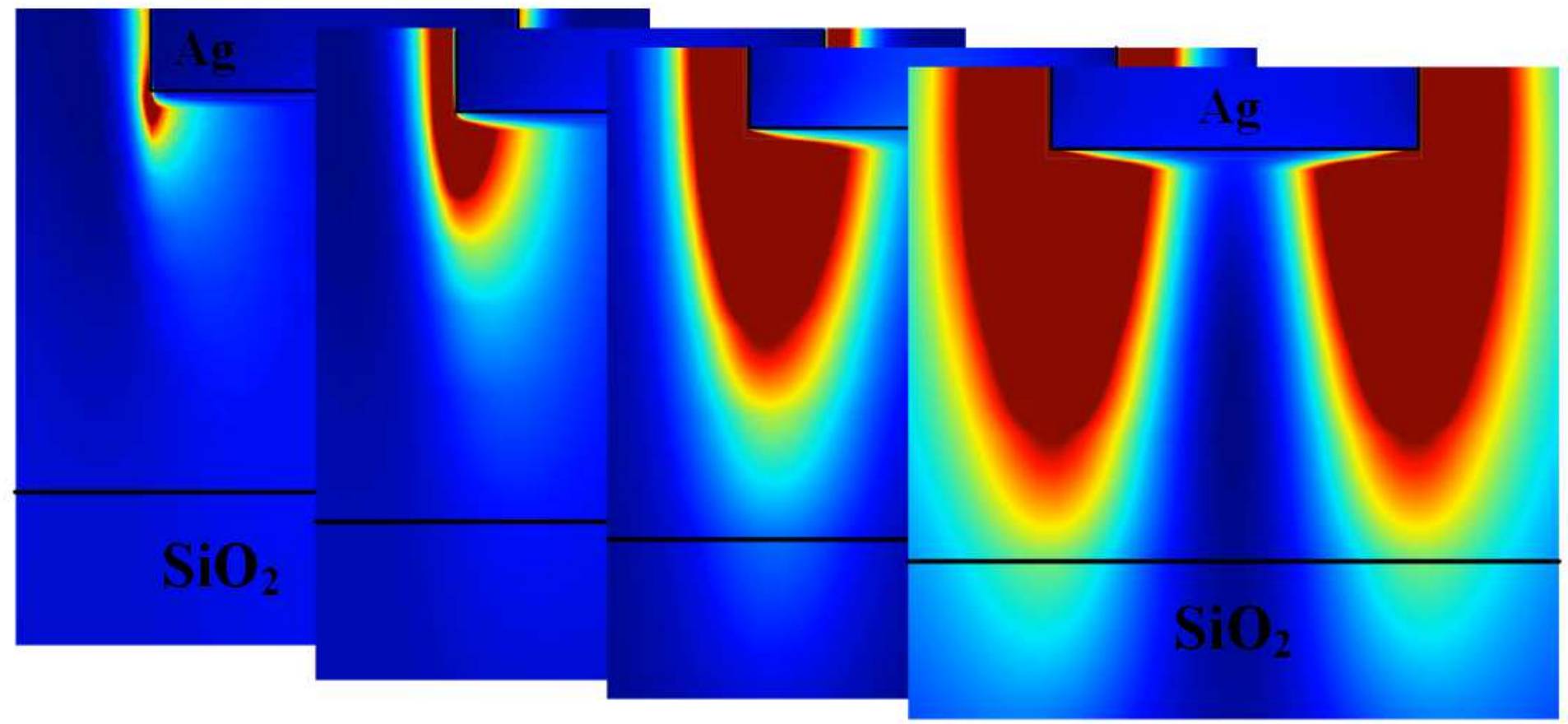

Figure 3

Electrical field profile, which is formed under elliptic nanoparticle. 


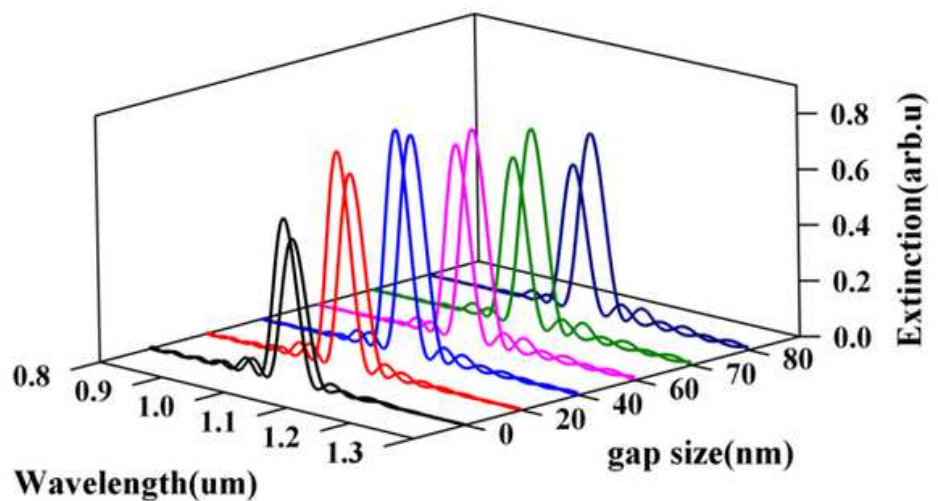

(a)

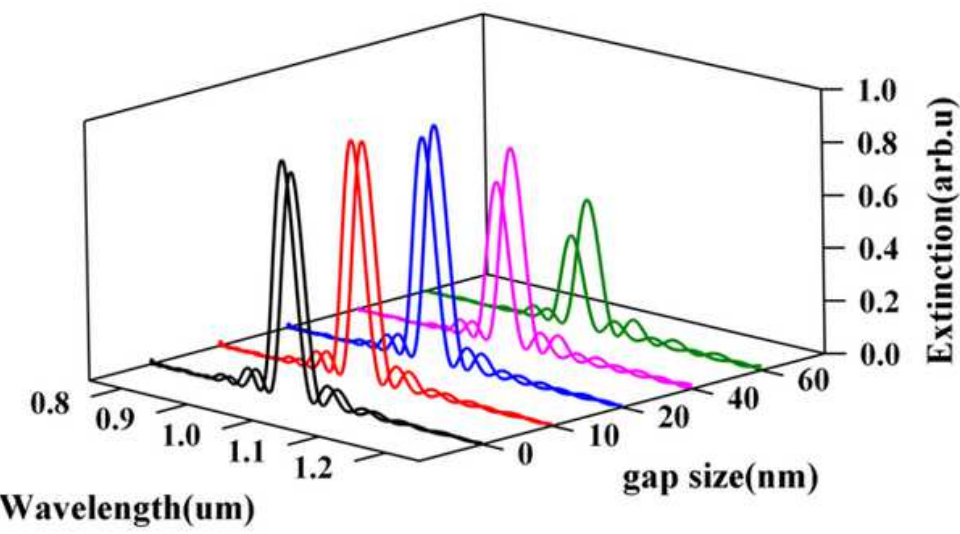

(c)

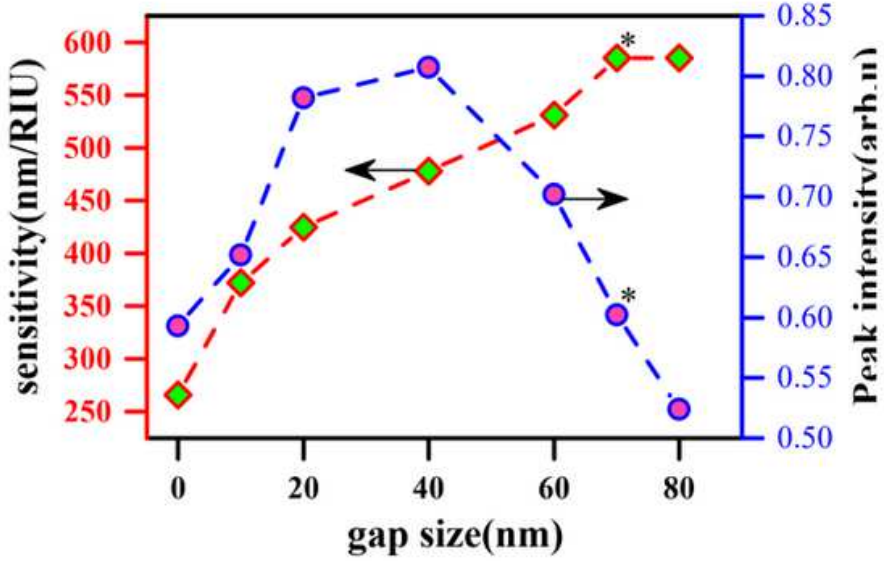

(b)

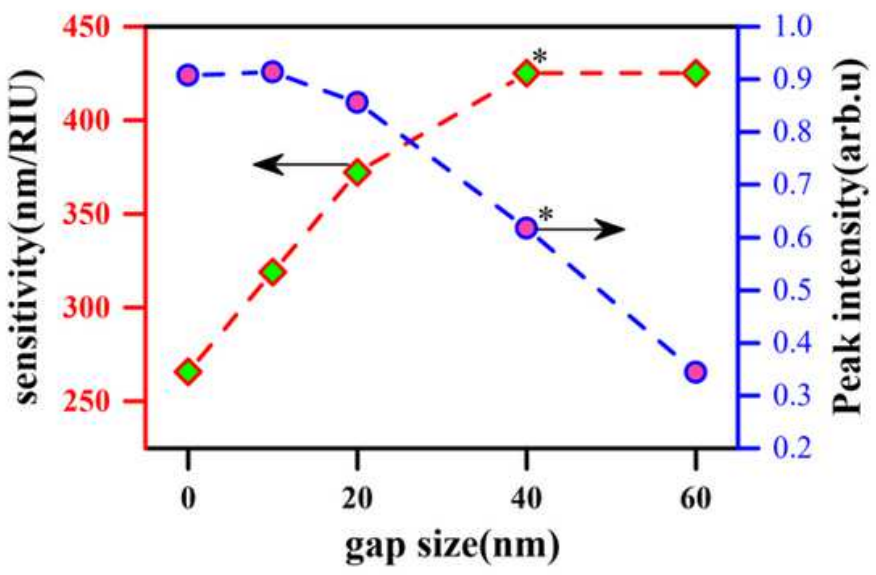

(d)

Figure 4

(a) The effect of gap variation for the Ag elliptic disc on extinction spectrum and (b) Sensitivity variation and normalized amplitude of extinction spectrum peak (c) The effect of gap change for the Ag circular disc on extinction spectrum and (d) Sensitivity variation and normalized amplitude of extinction spectrum peak. 


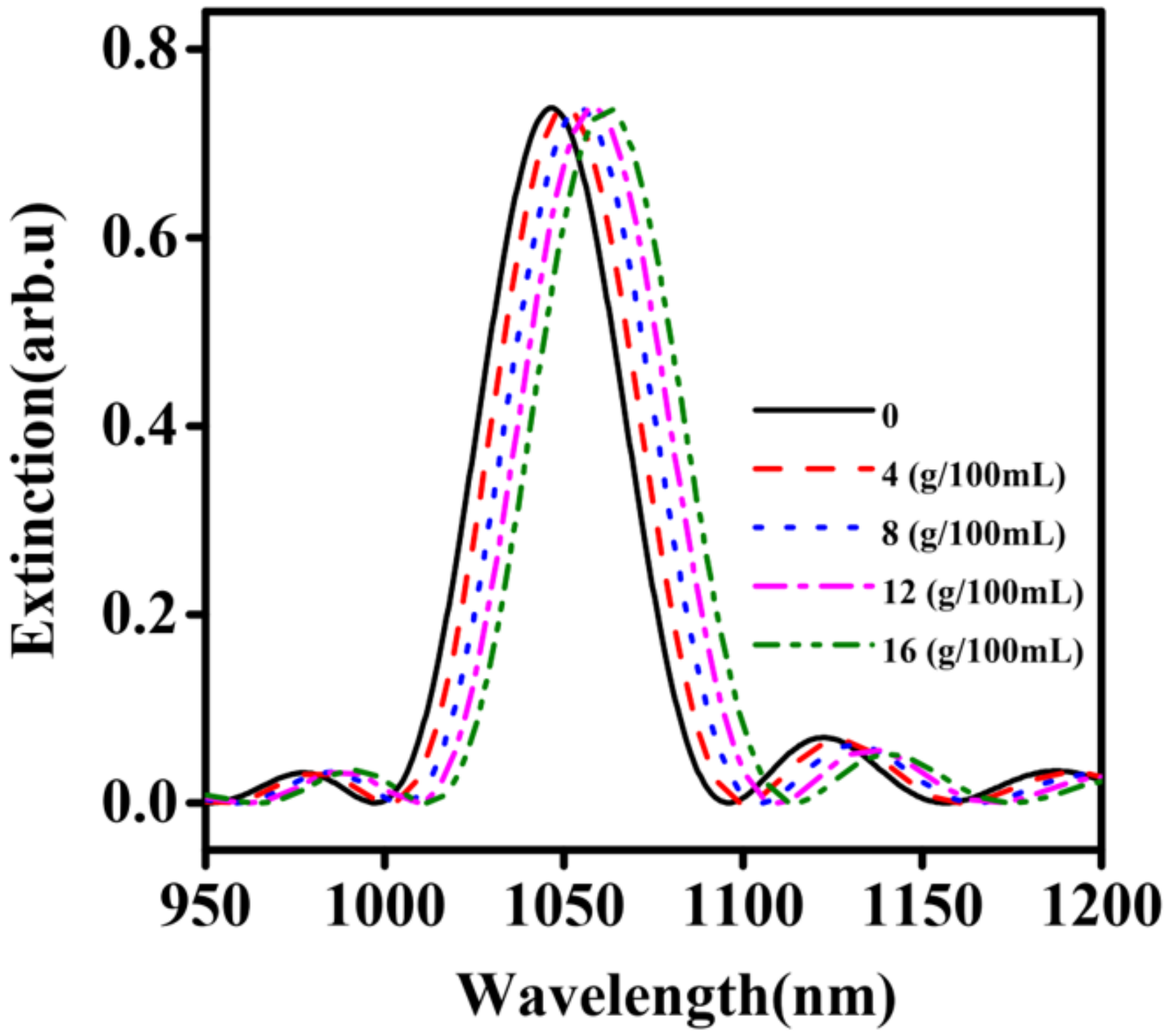

Figure 5

Extinction spectrums for different densities of glucose/fructose. 